\title{
Black esophagus: exploring the dark
}

\author{
Robert Forstera, Délcio Aparecido Durso ${ }^{a}$, Edoardo Filippo de Queiroz Vattimo ${ }^{\text {, }}$ \\ Luis Massuo Marutac ${ }^{c}$ Ana Luiza Werneck-Silvac, Vilma Takayasud, \\ Marcia Yoshie Kanegae ${ }^{d}$, Fabiana Roberto Lima ${ }^{\mathrm{e}}$
}

Forster R, Durso DA, Vattimo EFQ et al. Black esophagus: exploring the dark. Autopsy Case Rep [Internet]. 2013; 3(3): 41-48. http://dx.doi.org/10.4322/acr.2013.027

\section{ABSTRACT}

\begin{abstract}
Black esophagus is a rare but underdiagnosed disease. It occurs most frequently in severely ill patients and carries a high mortality rate. Cause of death is usually attributed to the comorbid conditions. Treatment is directed at the underlying cause, acid suppression and keeping the patient nil-peros. Surgery is needed in complicated cases and stenosis is the most feared longterm sequel. In the present article, two cases are described and literature is reviewed.
\end{abstract}

Keywords: Esophageal Diseases; Esophagitis; Critical Illness; Diabetic Ketoacidosis.

\section{CASE 1}

A 47-year-old male patient was admitted complaining of macroscopic hematuria and weight loss of $20 \mathrm{~kg}$ over the last two months. On physical examination bilateral massive abdominal masses were palpable extending from the flanks to the iliac fossae bilaterally. Laboratory tests showed marked metabolic acidosis, hyperkalemia and electrocardiographic alterations, which required prompt intravenous infusion of calcium gluconate and hemodialysis. Urinalysis showed pyuria. Abdominal ultrasound imaging and computed tomography scan revealed hepatic and renal polycystic disease. The kidneys were markedly enlarged extending from their original site until the iliac fossae and their architecture was effaced by multiple cysts. On the following days the patient suffered an ischemic stroke and a pulmonary thromboembolism. Outcome was characterized by daily fever despite of the use of broad-spectrum antibiotics and negative blood and urine cultures. A PET SCAN was performed which was compatible with infection of multiple and bilateral renal cysts. Therefore bilateral nephrectomy was performed, and because of technical difficulties adrenals were also excised. Surgery was technically challenging, the patient remained hypotensive and required packed red blood-cell transfusion. After ICU discharge the patient started experiencing epigastric pain and heartburn. Esophagogastroduodenoscopy (EGD) (Figure 1) revealed necrotizing esophagitis

\footnotetext{
a Department of Internal Medicine - Hospital das Clínicas - Faculdade de Medicina - Universidade de São Paulo, São Paulo/SP - Brazil.

${ }^{\mathrm{b}}$ Faculdade de Medicina - Universidade de São Paulo, São Paulo/SP - Brazil.

${ }^{c}$ Endoscopy Service - Hospital Universitário - Universidade de São Paulo, São Paulo/SP - Brazil.

d Department of Internal Medicine - Hospital Universitário - Universidade de São Paulo, São Paulo/SP - Brazil.

e Anatomic Pathology Service - Hospital Universitário - Universidade de São Paulo, São Paulo/SP - Brazil.
}

Copyright $\odot 2013$ Autopsy and Case Reports - This is an Open Access article distributed of terms of the Creative Commons Attribution NonCommercial License (http://creativecommons.org/licenses/by/3.0/) which permits unrestricted non-commercial use, distribution, and reproduction in any médium provided article is properly cited. 


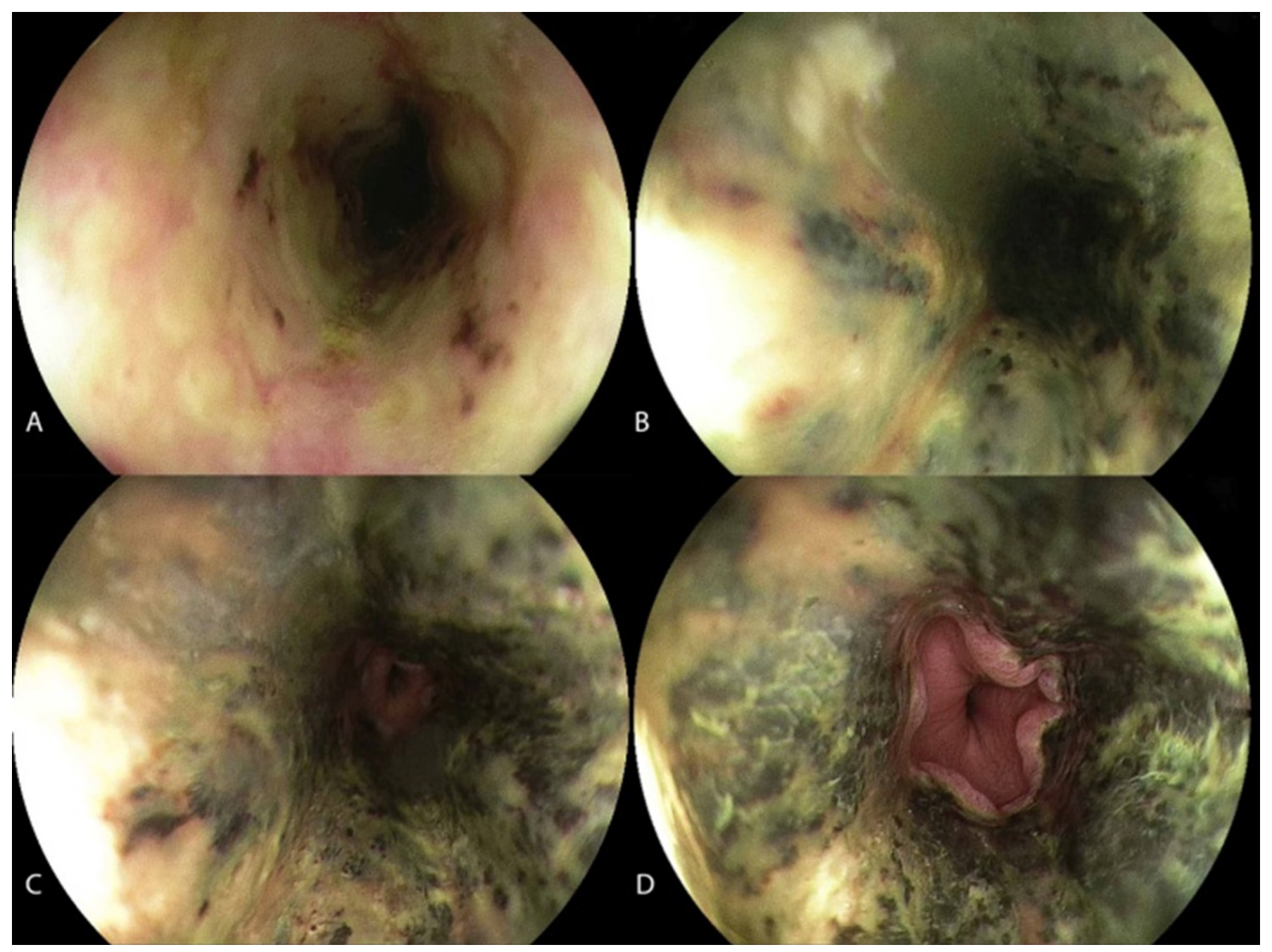

Figure 1 - Esophagoscopy from proximal (A) to distal (D) esophagus, showing acute necrotizing esophagitis. Black esophagus.

compatible with the endoscopic diagnosis of "black esophagus". The duodenal bulb was deformed by scarred ulcers and a small ulcer was visible in the anterior wall. Enteral nutrition, a proton pump inhibitor, a prokinetic drug and sucralfate were prescribed. A week later, control EGD showed an effacement of the blackened areas, which had been replaced by a diffuse fibrinous esophagitis. Recovery was uneventful since then. The patient went on hemodialysis and resumed oral feeding.

\section{CASE 2}

A 45-year-old male patient was admitted after a two-day history of dyspnea, odynophagia, nausea, vomiting, generalized weakness and drowsiness. On physical examination he was dehydrated, tachycardic, tachypneic, normotensive, afebrile, conscious but drowsy. He had been diagnosed with type 2 diabetes mellitus 5 years earlier but did not follow a regular treatment, giving up the use of the prescribed hypoglycemic agents during the last year. The initial laboratory workup was compatible with the diagnosis of diabetic ketoacidosis with a blood glucose of $469 \mathrm{mg} / \mathrm{dL}$ (reference value $(\mathrm{RV})<$ $100 \mathrm{mg} / \mathrm{dl}$ ), venous blood $\mathrm{pH} 7.14$ (RV; 7,35-7,45), bicarbonate $9 \mathrm{mEq} / \mathrm{L}(\mathrm{RV} ; 24 \mathrm{mEq} / \mathrm{l})$, and urinalysis showing marked ketonuria. Glycated hemoglobin was $15,1 \%$ (RV < 5,7\%) After metabolic control, the patient started complaining of heartburn and odynophagia. Esophagogastroduodenoscopy revealed friable esophageal mucosa, shallow ulcerations and extensive areas of mucosal necrosis along the medial and distal third of the esophagus consistent with the diagnosis of "black esophagus" (Figure 2).

Histopathological examination of the esophageal biopsy specimen showed a marked acute inflammatory infiltrate, foci of liquefactive necrosis, presence of rare bulky endothelial and stromal cells, foci of intravascular fibrin, lack of representation of stratified squamous epithelium, with dark brown granular material (Figure 3). The 


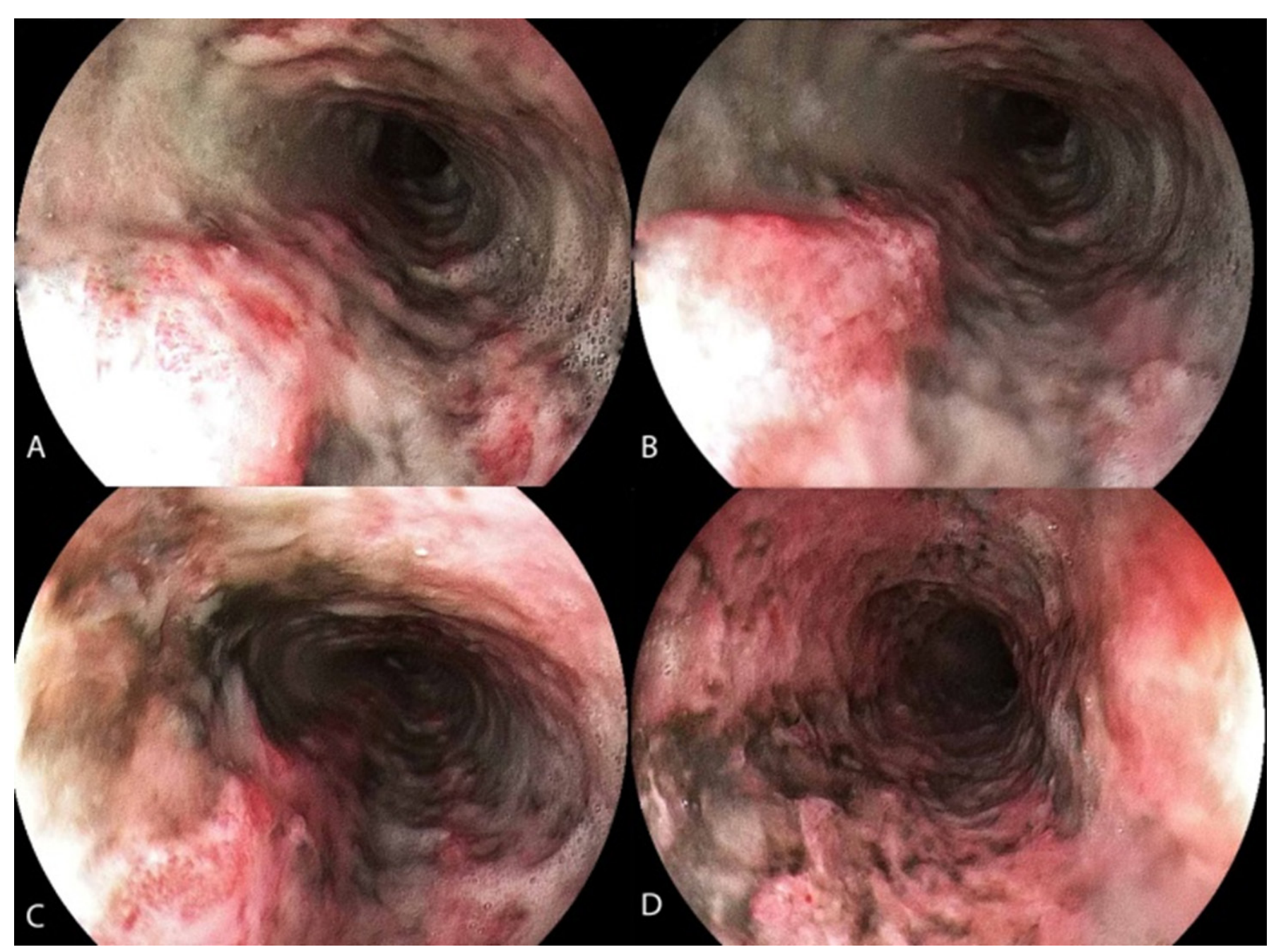

Figure 2 - Endoscopic view of the esophagus (A to $\mathbf{D})$ showing acute esophageal necrosis. Black esophagus.

Grocott stain was negative for the presence of fungi. Cytomegalovirus (CMV) and herpes virus were absent on immunohistochemical research. Serologies for HIV and CMV were negative. The patient was kept nil-per-os and prescribed parenteral hydration, proton pump inhibitor, sucralfate and fluconazole.

Esophagogastroduodenoscopy, undertaken on the third day, still showed darkened esophageal mucosa, now covered by patches of fibrinous material (Figure 4). The gastric mucosa was intact but a bulbar ulcer surrounded by scarred areas was present. On the sixth day another EGD was performed showing remission of the esophagitis as well as of the duodenal ulcer.

\section{DISCUSSION}

Acute esophageal necrosis (AEN), also referred to as "black esophagus" or necrotizing esophagitis, is a rare clinical entity, named by its characteristic presentation as a circumferential black-appearing necrotic esophageal mucosa on esophagogastroduodenoscopy (EGD).

A 2010 review of the literature disclosed only 112 cases, ${ }^{1}$ though black esophagus is likely an underdiagnosed syndrome. Large series of autopsies and reviews of esophagogastroduodenoscopies have estimated a prevalence ranging from $0,01 \%$ to $0,28 \% .^{2-4}$ There is a remarkable predominance among men, which are four times more commonly affected than women. Peak incidence occurs in the seventh decade, with the average age of 68.4 years. ${ }^{1}$ Generally, at least two comorbidities are present. ${ }^{4}$ Renal insufficiency, mostly acute (29$33 \%)$, diabetes mellitus (21-27\%), cancer (18-29\%) and hypertension (17-21\%) are most frequently associated. Duodenal ulcers $(25-33 \%)$ are a frequent concomitant finding. ${ }^{4,5}$ On diagnosis, other frequently associated clinical conditions are cardiac, pulmonary and renal disease, coagulopathy, ketoacidosis, cirrhosis, malnutrition, acute alcohol 


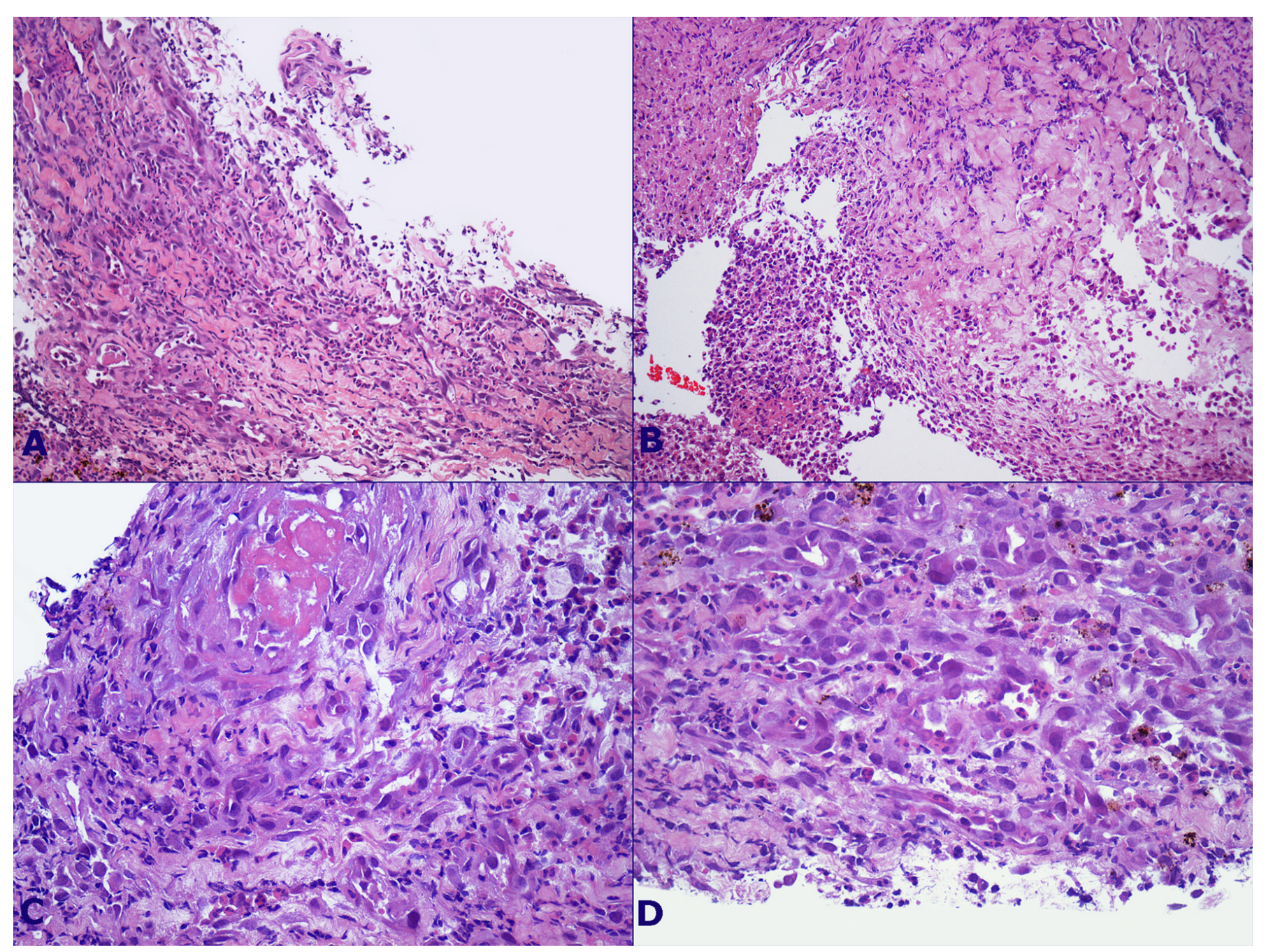

Figure 3 - Photomicrograph of the esophagus. A - granulation tissue of the ulcer bed; $\mathbf{B}$ - fibrinoid necrosis with countless polymorphonuclear leukocytes; C - acute inflammatory infiltration and thrombosed capillary within the granulation tissue; $\mathbf{D}$ - higher magnification of the ulcer bed showing neovascularization, reactive stromal and endothelial cells with enlarged nuclei.

intoxication, alcohol abuse, acute alcoholic hepatitis, peptic disease, acute fatty liver of pregnancy, acute pancreatitis and sepsis. ${ }^{2,6}$

The etiology of acute esophageal necrosis is multifactorial. Hemodynamic instability or lowflow states, impaired mucosal barrier and massive influx of acid gastric contents into the esophagus seem to be the main contributing factors. ${ }^{5}$ In some cases infection is superposed or may represent an additional contributing factor. ${ }^{3}$ Simultaneous presence of these insults seems to be necessary to trigger the development of acute necrotizing esophagitis. The important role of ischemia in the pathogenesis of black esophagus is demonstrated by the high frequency of concomitant malperfusion states, the association with duodenal ulcers and almost universal involvement of the distal esophagus (97\% of cases, ${ }^{5}$ which is the less vascularized third. It is supplied by branches of the left gastric and inferior phrenic arteries. ${ }^{7}$ The proximal two thirds of the esophagus are well vascularized, receiving its blood supply from branches off the inferior thyroid, bronchial arteries and vessels deriving from the thoracic aorta.

Significant vasculopathy as seen in diabetes mellitus, atherosclerosis, cardiovascular and renal diseases are frequent risk factors that may predispose to ischemic injury. Impaired tissue perfusion states, as observed during cardiac arrhythmias, congestive heart failure, systemic inflammatory response syndrome, such as in severe acute pancreatitis, lactic- and ketoacidosis, acute blood loss, trauma, hypothermia and shock are some of the conditions related to the ischemic compromise of the esophagus. Thromboembolic phenomena and hypercoagulability states, such as in solid or hematological malignancies, antiphospholipid antibody syndrome and atherosclerosis have also 




Figure 4 - Endoscopic view of the esophagus (A to D) showing partial recovery of the necrotic esophagitis, covered by patches of fibrinous material.

been implicated in the genesis of AEN. ${ }^{2,8-12}$ Cases of isolated esophageal ischemia are rare due to its rich arterial supply. Hence, ischemia seems to be the trigger event of AEN in the presence of impaired mucosal repair and augmented acid reflux. The latter is better explained by diminished mucosal buffering, disrupted local protective barriers and impaired defense mechanisms due to a variety of causes such as sepsis, malnutrition, acidosis, renal insufficiency, cancer, chronic pulmonary disease, acute or chronic decompensated diabetes mellitus, immunosuppressive states such as acquired immunodeficiency syndrome (AIDS) and other critical illnesses. Chemical damage due to acid reflux may be caused by gastric outlet obstruction as a consequence of edema and inflammation associated with duodenal ulcers or by transient gastroparesis due to alcohol intoxication, ketoacidosis, hyperglycemia and systemic inflammatory states. Postoperative recumbency, gastric volvulus, hiatal hernia, decreased intraesophageal luminal pressure and decreased lower esophageal sphincter resting pressure (as observed in cases of chronic alcohol abuse $^{6}$ ) represent other causes of increased acid reflux leading to injury of the vulnerable mucosa.

Both, impaired mucosal defense mechanisms and augmented acid content in the esophagus may lay the ground to ischemic injury and precipitate necrosis of the distal esophagus. Massive acid reflux impairs local blood flow autoregulation and thus limits the mucosal buffering and luminal clearance capacity, hence predisposing to ischemic and/or acid injury. Since the first description by Goldenberg et al., in 1990, oxyradical formation from ischemia and reperfusion injury is known to play an important role in the pathogenesis of black esophagus. ${ }^{13}$

Opportunistic infections in immunocompromised patients are not a rare finding. Herpes simplex virus I, Lactobacillus acidophilus, Candida albicans, Penicillium chrysogenum and cytomegalovirus were some of the isolated agents. 
On histology the esophageal necrosis may be restricted to the mucosa but may extend into the submucosa and muscularis mucosae. Fullthickness necrosis of the esophageal wall has been described in surgical and autopsy specimens. Abundant necrotic debris and spot-like or confluent hemorrhagic areas replace the viable epithelium. Acute inflammatory infiltrate, predominantly of neutrophilic granulocytes, has been described, but may be absent. Vascular thrombi and deranged muscle fibers are occasionally associated findings..$^{5,14}$

The most common clinical presentation is upper gastrointestinal bleeding represented by at least one of the following: hematemesis, melena or coffee ground emesis, accounting for up to $90 \%$ of the presentations in the largest reviews of literature on AEN. ${ }^{1,5}$ At the other end of the spectrum, asymptomatic presentation has been described. ${ }^{5,15}$ Less frequent symptoms include epigastric pain and dysphagia, nausea, lightheadedness and other nonspecific symptoms.

On esophagogastroduodenoscopy, AEN classically presents as a black-appearing circumferential necrosis of the mucosa in the distal third of the esophagus, usually extending up to the distal two thirds and eventually the entire esophagus. Characteristically, the esophageal necrosis is sharply limited by the Z-line and gastric mucosa is usually spared. Yellow exudates and a friable mucosa may be present. Duodenal ulcers are a frequently associated finding.

Black esophagus can be staged as follows: Stage 0 refers to a viable, pre-necrotic and normal appearing mucosa; Stage 1 represents the typical circumferential black friable necrotic mucosa sharply ending at the Z-line. In Stage 2, black spots and thick white, removable exudates cover the pink friable mucosa in a "chess-board" pattern. In Stage 3, the esophageal mucosa regains its normal appearance while on histologic analysis granulation tissue may still be present. Strictures and stenosis may occur as sequelae in stages 2 and 3 . Stage 3 may occur as early as 1 or 2 weeks from initial presentation. ${ }^{1,5}$

Cultures for virus and bacteria, as well as fungi should be ordered on endoscopically biopsied material, particularly in immunocompromised individuals, to exclude an infectious etiology or superimposed infection. ${ }^{5}$
Differential diagnosis includes pseudomelanosis, melanocytosis, coal dust deposition, malignant melanoma and acanthosis nigricans. Distinction of those entities from AEN is easily made by the absence of true necrosis and ulceration in the latter.

For diagnosis of acute esophageal necrosis, ingestion of caustic agents should be excluded by history and absence of oropharyngeal findings. ${ }^{16}$

Up to $10 \%$ of patients have been reported to experience esophageal strictures or true esophageal stenosis, which configure the most common complication. ${ }^{5}$ Perforation and mediastinitis are other common complications. In the largest literature review, mortality rates ranged from 3a, $8 \%$ to $38 \% .^{1}$ Nevertheless, only approximately $6 \%$ of cases were directly related to the black esophagus syndrome, but rather to the usually severe comorbid conditions. ${ }^{1,3-5}$ Autopsy disclosed black esophagus as the immediate cause of sudden death in a patient with mental retardation and a history of alcohol abuse.${ }^{14}$ In other sudden death autopsy cases, AEN has been related to fatal upper $\mathrm{GI}$ hemorrhage, but in these cases it was not clear whether comorbidities were present. ${ }^{17,18}$

The mainstay of treatment of black esophagus is correction of the the underlying cause, hydration, re-establishment of the patient's volemic status and correcting anemia. Parenteral nutrition may be needed in malnourished patients, while passage of a nasogastric tube should be avoided due to the risk of perforation. Treatment further includes keeping the patient nil-per-os, full-dose proton pump inhibitor or $\mathrm{H} 2$ antagonist therapy associated with sucralfate. $2,4,5$

The use of antibiotics in AEN is controversial. While antibiotics have been associated with necrotizing esophagitis in a few reports, ${ }^{19}$ empiric antimicrobial treatment should be promptly started in rapid clinical deterioration, suspected esophageal perforation, mediastinitis and unexplained fever. Immunosuppressed patients, such as AIDS patients, cirrhotics, transplant recipients or patients in dialysis should be highly considered to receive antibiotics. ${ }^{2}$

Surgical treatment is mandatory in cases of esophageal perforation, mediastinitis and abscess formation. After the healing phase, surgical treatment may be necessary in stenosis refractory to endoscopic dilation attempts. 
Case 1 refers to a 47 year-old patient which had bilateral nephrectomy because of infected polycystic renal disease. This patient received broad-spectrum antibiotics and hemodialytic treatment. During the postoperative period, he started complaining of epigastralgia, pyrosis and refused oral feeding.

Probably due to the severity of the underlying disease, acute esophageal necrosis was not diagnosed since the beginning, once EGD showed a typical stage 2 injury. The middle and distal esophagus were affected by necrotic mucosa and alternating white exudates (Figure 1). This endoscopic pattern is called "chess-board" due to its appearance. In the same figure the sharp limit between the necrosed esophageal mucosa and the normal gastric mucosa denotes the richer arterial supply and acid-resistance of the gastric mucosa. Renal failure, the most frequent comorbidity present in patients with black esophagus was present in this patient. It has been postulated that acidosis impairs local blood flow regulation. Severe infection and hypotension may also have been involved in homeostasis derangement. Prolonged decubitus and postoperative gastroparesis may have contributed to acid reflux into the esophagus. Hemodynamic instability and the inflammatory state may have been the precipitating factors for injury to a dysfunctional mucosa. The presence of duodenal ulcers on initial EGD, another frequent simultaneous finding in $A E N$, reenforces the hypothesis of hemodynamic instability, systemic inflammation and fasting as precipitating events. Whether the ischemic stroke and pulmonary thromboembolism and related alteration of blood coagulation contributed to the pathogenesis of black esophagus is speculative. After treatment of the associated conditions a new EGD revealed fibrinous patches, effacement of the black areas and friable pink mucosa. This reminds us of the amazingly fast and efficient regeneration capacity of the esophageal mucosa after the offending factors have been reversed.

Case 2 refers to a 45 year-old male patient with type 2 diabetes, who presented to the emergency room with repeated vomiting, severe dehydration and hyperglycemia.

The diagnosis of diabetic ketoacidosis was made. Acute diabetic gastroparesis and dysfunction of the lower esophageal sphincter due to glucose toxicity contributed to stasis and acid reflux. Associated relative ischemia due to dehydration and immune dysfunction demonstrated by the patient's elevated glycated hemoglobin ( $\mathrm{Hb} 1 \mathrm{Ac})$ levels, seemed to have contributed to necrotic injury of the esophagus in this acutely ill patient. Diabetes mellitus and diabetic ketoacidosis are two frequently cited comorbid conditions in patients presenting with AEN. The proposed impairing effect of acidosis on arterial blood flow regulation in the esophageal wall may have been an additional factor predisposing to necrotizing esophagitis. Repeated vomiting could have been a possible precipitating factor in this case.

Due to the multitude of symptoms present in diabetic ketoacidosis, which overlap with those of black esophagus, attention was drawn to the esophageal disorder only after stabilization and reversal of ketoacidosis. This may be one of the causes of underdiagnosis of AEN.

In this case difficult distinction between necrotic debris and candidiasis led to addition of oral fluconazole to the standard therapeutic regimen.

Histologic analysis of the biopsy specimens showed typical findings in early black esophagus. Microvascular thrombosis, observed in this case and described in previous reports, may have been a consequence of endothelial dysfunction of diabetes mellitus. Neovascularization, reactive stromal and endothelial cells with enlarged nuclei also found in this case are early regenerative changes (Figure 2 ). The therapeutic regimen adopted in this case led to symptomatic control and near-normalization of the endoscopic findings in 48 hours. In view of the distinct possible appearances during the healing process, maybe subdivision of the proposed staging system $^{2,5}$ in an early $2 \mathrm{a}$ and a late $2 \mathrm{~b}$ stage without necrosis, as follow-up EGD revealed in this case, could be useful.

The outcome in this case corresponds to the majority of the cases when the offending conditions are reverted and appropriate treatment is adopted.

In conclusion, AEN is a rare and underdiagnosed disease. Critical illness and multiple comorbidities are usually associated. It may be interpreted as a sign of poor prognosis, as mortality rate is up to $38 \%$. Cause of death is usually attributed to the comorbid illness, but deaths due to black esophagus have been reported. Treatment is directed at reversal of the underlying cause, acid suppression and keeping the patient nil-peros. Further research is needed to clarify the exact involved pathophysiological mechanisms. 


\section{REFERENCES}

1. Day A, Sayegh M. Acute oesophageal necrosis: A case report and review of the literature. Int J Sur. 2010;8:6-14. http://dx.doi.org/10.11604/pamj.2013.14.109.2000

2. Gurvits GE. Black esophagus: acute esophageal necrosis syndrome. World J Gastroenterol. 2010;16:3219.

3. Augusto F, Fernandes V, Cremers MI, et al. Acute necrotizing esophagitis: a large retrospective case series. Endoscopy. 2004;36:411. http://dx.doi.org/10.1055/s-2004-814318

4. Grudell ABM, Mueller PS, Viggiano TR. Black esophagus: report of six cases and review of the literature, 1963-2003. Dis Esophagus. 2006;19:105-10.

5. Gurvits GE, Shapsis A, Lau N, et al. Acute esophageal necrosis: a rare syndrome. J Gastroenterol. 2007;42:29. http://dx.doi.org/10.1007/s00535-006-1974-z

6. Hong JW, Kim SU, Park HN, Seo JH, Lee YC, Kim H. Black esophagus associated with alcohol abuse. Gut Liver. 2008;2:133-5. http://dx.doi.org/10.5009/gnl.2008.2.2.133

7. Patti MG, Gantert W, Way LW. Surgery of the esophagus. Anatomy and physiology. Surg Clin North Am. 1997;77:959-70.

8. Cappell MS. Esophageal necrosis and perforation associated with the anticardiolipin antibody syndrome. Am J Gastroenterol. 1994;89:1241-5.

9. Odelowo OO, Hassan M, Nidiry JJ, Marshalleck JJ. Acute necrotizing esophagitis: a case report. J Natl Med Assoc. 2002; 94:735-7.

\section{Conflict of interest: None}

Submitted on: $03^{\text {rd }}$ August 2013

Accepted on: $10^{\text {th }}$ September 2013

Correspondence: Departamento de Clínica Médica Hospital das Clínicas da Faculdade de Medicina da USP Av. Dr. Enéas Carvalho de Aguiar, 255 - São Paulo/SP - Brazil CEP: 05403-000 - Phone: +55 (11) 2661-6300

E-mail: robertforster.ufrj@gmail.com
10. Hawari R, Pasricha PJ. Esophageal infarction. Curr Treat Options Gastroenterol. 2007;10:57-60.

11. Yasuda H, Yamada M, Endo $\mathrm{Y}$, Inoue K, Yoshiba M. Acute necrotizing esophagitis: role of nonsteroidal antiinflammatory drugs. J Gastroenterol. 2006;41:193-7. http:// dx.doi.org/10.1007/s00535-005-1741-6

12. Burtally A, Gregoire P. Acute esophageal necrosis and lowflow state. Can J Gastroenterol. 2007;21:245-7.

13. Goldenberg SP, Wain SL, Marignani P. Acute necrotizing esophagitis. Gastroenterology. 1990;98:493-6.

14. Tsokos M, Herbst $\mathrm{H}$. Black oesophagus: a rare disorder with potentially fatal outcome. A forensic pathological approach based on five autopsy cases. Int J Legal Med. 2005;119:14652. http://dx.doi.org/10.1007/s00414-004-0509-5

15. Rejchrt S, Douda T, Kopácová M, et al. Acute esophageal necrosis (black esophagus): endoscopic and histopathologic appearance. Endoscopy. 2004;36:1133. http://dx.doi. org/10.1055/s-2004-825971

16. Gumaste VV, Dave PB. Ingestion of corrosive substances by adults. Am J Gastroenterol. 1992;87:1-5.

17. Tsokos M. Black esophagus. Forensic Sci Med Pathol. 2011;7:374-6.

18. Jacobsen NO, Christiansen J, Kruse A. Incidence of oesophageal necrosis in an autopsy material. APMIS. 2003;111:591-4. http://dx.doi.org/10.1034/j.16000463.2003.1110509.x

19. Mangan TF, Colley AT, Wytock DH. Antibiotic associated acute necrotizing esophagitis. Gastroenterology. 1990;99:900. 